\title{
You never know: the journey into convincing ourselves
}

\section{Edgard Cornacchione}

Ave. Prof. Luciano Gualberto, 908,

(Bldg FEA3, office 223),

Sao Paulo, SP, 05508-010, Brazil

Email: edgardbc@usp.br

\begin{abstract}
My argument is that every decision, regardless of size, relevance, opportunity or consequences, could be traced back to a single mind. Concrete action triggered by a decision-making process rises from a multitude of elements chained together, capable of influencing or convincing an individual's process, with emotional and rational dimensions, adding critical elements to that person's existing frame of reference, experience and knowledge. Making sense of data requires resources, in both analogue and digital formats, as a complex process, difficult to explain, but present in everyday decisions. Acknowledging and adopting a set of resources to improve such process represents a natural way to improve quality, leaving less room for destiny or luck. One may argue that the path to a bolder life, including happiness, is rich in judgment, and associated skills are in high demand. Fostering such skills and the technological support represents a big leap forward towards this journey.
\end{abstract}

Keywords: machine learning; decision; analytics; organisational intelligence; artificial intelligence.

Reference to this paper should be made as follows: Cornacchione, E. (2016) 'You never know: the journey into convincing ourselves', Int. J. Multivariate Data Analysis, Vol. 1, No. 1, pp.96-105.

Biographical notes: Edgard Cornacchione is a Full Professor at the University of Sao Paulo (Brazil). A Brazilian Chartered Accountant, he holds a PhD in Accountancy (USP/Brazil) and a PhD in Education (UIUC/USA). He published many studies and books with a record of successful grant application and funded projects. He acts as editorial board member and reviewer for journals and conferences in the USA, Europe and Brazil. His research interests gravitate around repositioning accounting in relation to long term sociotechnical disruptions with large impact on business and professional capacities, in both academic and corporate environments, including effects of advanced technologies.

"Education is the path from cocky ignorance to miserable uncertainty." (Mark Twain)

\section{Acts, facts, right or wrong ... with a twist of emotion}

Acts and facts are all over the places. Organising acts and facts as evidence and into meaningful elements of a decision-making process is a different thing, being able to identify usefulness in evidence takes time, training and mistakes. Reasoning is often 
presented as the right way of informing our decisions. On the other hand, emotion is less explored and tends to operate in this decision realm, but in another dimension. As scissors have two blades, we could argue that decisions are made in an interesting combination of reason and emotion (Damasio, 1996; Lowenstein and Lerner, 2003). On extremes we will find an intense scientific debate igniting claims of decisions heavily driven by emotion, or claims of a fully rational decision-making process (Lerner et al., 2015).

For instance, in a typical daily routine, you may decide about a restaurant to have a meal. Walk into it, order your meal, enjoy it, pay for it and then leave. From the standpoint of the consumer it seems like a simple task, however even for those not interested in many details, it is easy to observe the amount of decisions to be made, all supported by information in different stages and formats. In addition, the slightest input in this process (even at random) could have enough power to change the main outcome of the process. Sure, both emotion and reason play their parts. Once we assume another point of view, for instance, the provider, decisions are also piling up and a set of elements of concern is very clear. No meal would be available without proper planning. Seeing ahead of time, assuming conditions, making bets about supply and demand, even guessing, are all part of the provider's decision chain. Results, in many formats, are basically a consequence of proper alignment between provider and consumer while being products of their emotional and rational dimensions, combined.

Now, forget about the typical local cozy restaurant that must have appeared in your mind and transport your thinking to the West Lake Restaurant (Changsha, China), with about 5,000 seats, or to the Damascus Gate Restaurant (Damascus, Syria), with over 6,000 seats. Think again about the costumer decision. Choosing to have a meal there, may engage a great deal of emotion and a great deal of reasoning. We could prepare a large list of items to support both dimensions. On the other hand, as the provider (the restaurant), being able to care for all clients while offering a memorable experience, takes another level of efforts. It is hard to consider all the facts and evidence that typically bloom every single day in such operation. Similarly, it is simple to accept the amount of information that is missed, disregarded, unused, although full of value and relevance to improve the quality of all decisions involved.

Beyond focusing on emotional or rational components of the decision-making process, I would like to make a point for putting more effort in considering what is being left behind, for ages, in many distinct industries, as a result of not paying enough attention, or for the sake of said technological limitations. How business, as providers, around the world, in many industries, would perform with a stronger consideration of evidence that has been historically missed or disregarded? How society, as clients, would behave with more and bolder elements combined and driving decisions?

From Gibson's affordance theory, dealing with clues in the environment that indicate possibilities for actions (Gibson, 1977), to affordances of technology, more specifically, the literature accepts that evidence contains potential to influence decision. The point now, being the improvement of technological affordance yielding more (both quantity and quality) evidence to support the aforementioned extremes of rational and emotional inputs to the decision-making process. In other words: we now have more potential to unveil more evidence, with greater quality, from acts and facts, faster.

When considering Campbell's experimenting society (Campbell, 1998) one may argue about utopia, however it is a clear structure inspired by the extreme rational decision-making process, backed by the golden standard in scientific method: the 
experiment. Looking twice, one may see beyond this, identifying opportunities to overcome the rational-emotional axis and focus on the evidence-gathering process, not only from a timeframe basis (ex ante), but from a manipulative and interventionist approach (specific content, cause). Randomised experiments can, in fact, afford a multitude of powerful causal claims while relying on a myriad of unobtrusive interventions up to more noticeable ones, directly affecting decisions.

\section{Fight or flight}

Human evolution is deeply rooted in decisions. Fear plays an important role, as we drive our decisions also based on our estimates of outcomes against conditions and potential. All these elements, without exception, come to our mind and body as information (sensed, interpreted) of all kinds. The ability to identify and treat them is a powerful proxy of the decision quality. Back to the fear, this distinguishes people as more or less inclined to risk in a given situation, adding to the beauty of the subject of decision making. It is foundational to establish the discussion about data and information on the stream of decision. It is, indeed, a quest for meaning. The individual trying to come to a decision or to reach a decision (both verbal constructions implies that it is 'out there') is dealing with a struggle, the quest for meaning, which involves content, communication channel, sender, receiver, history of the channel, existing conceptual assets, just to mention a few.

In our evolution, time makes difference. At the beginning, the set of statuses and risks was very distinct than what we have today (you may transfer this to any given condition or decision making scenario for the sake of comprehension). As we move up in time, statuses and changes become more complex, marginally. Mapping and sensing the environment requires more power as such environment is larger and deeper, in complexity. Thus, we humans developed learning abilities to help us transform and develop aiming at our own success in such environment, regardless of what we may agree about success.

It is clear that we, humans, chose to accept and develop skills associated with better quality decisions, impacting quality of our existence. Sorting out a number of acts and facts, making sense of them, in a timely fashion, became part of our being. Of course, not only ours, but, to an extent, we are learning how to cope with new and more complex situations, adding elements to our existing living structure: in other words, developing and using tools to support this process. And it seems that we still have a chance to keep succeeding at it.

Our daily routine or our organisations' set of transactions (i.e., the mentioned restaurant examples) are both highly complex nowadays, and usually referred to as part of the information revolution, challenging us while also simplifying our existence.

\section{Just do not jump into conclusions yet}

Assertively dealing with massive amounts of data (an inherited condition of any newborn these times) has become an integral part of our lives. As claimed before, this represents a skill, a highly demanded one. People will tackle this in different ways, according to the environment and existing frames of references (again, evolution). A critical element to 
pay attention (or to avoid) is the one often labeled as 'luck', or just error, for those leaning more towards the rationality side. How to avoid it? Or for the sake of a more precise meaning, how to reduce such room for error?

Time is directly involved in decision-making process, while risk can be related to time in decision making, and is a typical attribute of information (i.e., opportune). So, a way of reducing the room for error is using time in a proper fashion. Not too much, not too little. Finding this optimal point is what put us back into the game (i.e., fight or flight). Heuristics can be mentioned as a way of simplifying decisions that are somewhat similar in nature and artifacts may be generated to encapsulate heuristics, aiming at more complexity.

But people learn to cope with the process frequently adopting models, or simplified versions of the environment, players and attributes. Assuming that experiences and results can and will be shared, we develop strategies based on mirroring, rooted on vicarious learning and social development, as discussed by Vygotsky and Bandura. However, it seems vital to construct meaning of a decision, not only mimic it (no matter how effective it may be), for the sake of improving results in a dynamic environment.

Not only the decision-process must be continuously improved but, specially, the time-related efficiency linked to additional artifacts (or human extensions). One cannot afford to jump into conclusions too soon, or too late, risking the overall decision quality.

I will now bring a situation to shed light into this: the go/no-go decision in aviation during take-off. The relevance of human factors in the field of aviation is well documented (Wiegmann and Shappell, 2003), representing an essential variable when discussing related decision-making process and its consequences, despite all avionics. The typical scenario I would like to paint here is a plane, cleared for takeoff, taking up speed on a runway. This represents a decision scenario (go/no-go) that an individual (even not a pilot) experiences in every flight. The key is to sort out all variables in play, regarding, for instance, aircraft conditions and limits, engines, rotation speed, traffic, runway conditions and remaining distance, to describe some. The pilot, aiming for rotation, must check for a specific refusal speed $\left(\mathrm{V}_{1}\right)$ and balance whether there is still enough runway for a stop, while assessing all variables that would lead to a smooth take-off. The pilot, in other words, must assess many variables, against time, and find out if it is safe to continue with the take-off: the point-of-no-return, in layman's language. The regulation addressing a rejected takeoff (RTO) states: "pilot's initiation of the first action to stop the airplane during the accelerate-stop tests" (Office of the Federal Register, 2012). A big human decision supported by many artifacts (including high end avionics), but still a big human decision. In this sense, regarding on-board technology, possibly including decision support, the NTSB (2008) also recommended that the FAA "actively pursue with aircraft and avionics manufacturers the development of technology to reduce or prevent runway excursions and, once it becomes available, require that the technology be installed". At least, two things are worth mentioning here, linked to timely decision making: human role and artifacts (support systems).

\section{From educated guess to truly informed decision}

Hire 1,000 associates? Dismiss 1,000 contractors? Buy one or two pieces of heavy machinery? Purchase 5,000 tons of reagents or 4,998? Add available land to expand the current property or invest in another location? Sell a batch to a premier costumer at 
$\$ 1.00$ or $\$ 0.99$ per unit? Level up the average costumer acquisition cost by $\$ 1.00$ or $\$ 1.14$ ? Purchase an economy batch from premier provider at $\$ 1.00$ or develop a new provider with $\$ 1,000,000$ ? Ship the new model to 1,000 retailers according to expected consumer demand? Avoid a particular market in China and Italy according to social media trends? Revamp overall prices in $4 \%$ due to new market trends?

Moving from routine second-skin decisions to more complex ones that may lead to chained consequences in the business environment (such as the ones just mentioned in the prior paragraph); we can easily fade away from a solid pattern or structure. Of course, there are many well-structured decisions within the business world that creating algorithms capable of signalling important information or even triggering actions has been a reality for decades.

However, the more we move away from the known 3-M model (man, money and machine) into new terrain of business transactions and asset monetisation, the more we need to improve ways of tackling less structured decisions, envisioning non-existing paths. Yes, we have been learning to deal with sociotechnical advances, whereas massive innovation including severe disruption comes at a price.

Again, humans may take huge advantage of going beyond biological limits. This is not just a step into human enhancement, augmentation or Kurzweil's singularity, but a sound acknowledgement of currently accepted computer-based human expansion: a slow and steady switch from Bloom's lower order to higher order thinking. A businessman in the Renaissance could probably keep track of major business variables, but how does that relate to modern times? Even the smallest shop, currently, can take enormous advantage of business computer systems, at a cost-efficient level. And this not only at the memory level (information storage) but, mainly, at the process level, what was once regarded a sole task of human cortex (Kurzweil, 2012).

The transition is in place, with technology assuming an ever-increasing part in the information processing, especially in business (including less and less structured decisions). This trend is promoting a shift in manpower demand, from typical number crunchers to more avid talents with potential to perform on first-of-a-kind challenges. Here lies a societal change affecting work, professions, education, life style, success and happiness.

All the decision examples presented at the beginning of this topic imply specific education and accepted supporting framework. The problem is to frame the question, to anticipate the need for a decision, in a complex environment full of variables and contingencies. The problem is to do this millions of times per cycle, for structured operations or several times per cycle, for more complex and less structured ones. In this area, I like to adopt the overarching concept of opportunity costs. Choosing to abandon a decision in spite of other tends to stimulate powerful reflections. Technology also offers a valuable support to humans, here.

Moving from educated guess to truly informed decision requires meaningful storage (collection), access (extraction) and use (analysis) of data, not to mention the required network infrastructure and user access (i.e., smart devices). Universities and organisations have been investing massive amounts of resources (including financial) into data management for a long time. Data warehousing and mining initiatives could be recalled as a big step in the recent past, while big data and data analytics are current taking up speed in the business world. Business intelligence and analytics (BI\&A) appears as a new focus area (Chen et al., 2012), experiencing data evolution from structured relational databases, to web-based unstructured content to mobile and 
sensor-based content. Thus, it is clear that the organisational focus is shifting from the cold database size to its smart use (George et al., 2014), or inventive processing capabilities. Going beyond the $3 \mathrm{~V}$ model (volume, variety and velocity), big data embeds relevant concepts influencing the future of business. These capabilities would lead organisations to stronger business acumen and deeper strategy-oriented decisions, empowering business professionals, while setting up a future-facing role in companies. The new professional role (and attributes) is a major claim of the 2013 joint report by IMA (The Association of Accountants and Financial Professionals in Business) and Association of Chartered Certified Accountants (ACCA).

\section{Organisational intelligence}

I rely on five signals of change in business to help me develop this reflection: businesses are becoming:

a bigger (massive-base and flow)

b autonomous (awareness, decision and action)

c smarter (affordance and innovation)

d empathic (responsiveness and anticipation)

e more than money (motivation and drive).

This transformation is affecting small companies and large organisations as well. I am not developing these thoughts here, but using them to justify my claim that being able to create a knowledge environment (regardless of the industry) is key to support the information needs of organisations. Moreover, moving beyond the human edge is just necessary.

Knowledge is still key. However, there was a time when we could address business intelligence linking it solely to human capital. People, executives, managers, and associates, used to be the sole bearers of intelligence propelling business. Over time these people learned to rely more and more on computational power, on technology. But, at first, it was just rough computing. Now, it is different due to better affordance, in all senses, and we need to pay more attention to it when making sense of data.

Linking people to business is a hard task, linking people to business metrics, an even harder one. At the core of human resources development (HRD), learning and development (LD) are today even more integrated with business (Pease et al., 2014). Investing in people, once used to receive a clear training perspective, but today it is different. Accepting learning organisation and knowledge management ideas, one will soon link Business Intelligence to humans and technology, or even better, to enhanced humans. Talents in organisations may learn, and so may machines. Learning tools that empower people may rely in data and knowledge instruments that are just part of the set suggested for machine learning. Combining both worlds brings an interesting potential to be released. Data analytics must be considered in these two dimensions: what humans can do best and what computers can do best. Emphatically pursuing integration of such dimensions translates into a powerful business competitive edge. 


\section{World of data}

Sumerians, back 5,000 years in Mesopotamia, were dealing with a sexagesimal system (60-base), which was in pace with the complexity of that time. Counting and recording transactions were basic requirements for proper business management. They relied on a creative solution (zero would still take time to be 'invented'), evolving to a positional writing (Kaplan, 1999). Back to present time, transactions also evolved and, currently, business complexity is other.

Similarly, either Drucker's "what gets measured gets managed" or Deming's "if you can't measure it, you can't manage it" brings attention to data. However, Deming challenges management with 'the important stuff cannot be measured'. This is another path to what I have already mentioned here, the partial reach of rational and structured decision making. Decision making can be traced to utility, but utility is a function of the subject. In other words, individuals tend to weigh differently a similar set of variables in a particular context. Knowing the 'colour' and 'temperature' of such variables in a given situation is important. By colour and temperature I mean anything that can affect cold-data, in a timely manner (i.e., think of social media trackers). Just check Knack.it, for instance (big data in action for hiring).

All knowledge and expertise in collecting, extracting and analysing data, in alignment with decisions, are welcome, regardless whether it is coming from humans or machines. Redesigning how we prepare and develop professionals and systems represents a critical step towards this goal. As a transition, we may still rely on existing analytic techniques while creating the next ones.

\section{$7 \quad$ Is the cat dead or alive?}

Way back, Clarke (1980, pp.1-2) mentioned the pocket electronic library "a library not only of books, but of films and music...such a device could have enough memory to hold all the books in the world". It seems that ubiquitous data repository was a challenge well tackled by now. Making sense of amounts of data is another one. Relying on technology to achieve it seems wise: a review of leading papers on AI journals shows that.

Dreyfus and Dreyfus (1986) explored, from an artificial intelligence (AI) standpoint, five stages of skill acquisition, involving structured (mathematical manipulations, puzzles, delivery truck routing, petroleum blending) and unstructured (management, nursing, economic forecasting, teaching, and all social interactions) areas of decisionmaking:

a novice

b advanced beginner

c competence

d proficiency

e expertise.

The authors define the last stage as intuitive and involved, while all the preceding others are analytical and more detached. This move is important: expanding not only our 
memory, but our processing abilities, as well, in an integrated (involved) way. Processing in light of a purpose, for the sake of learning, as making sense of data and information.

AI, or as coined by McCarthy in 1955, "the science and engineering of making intelligent machines" (McCarthy, 2007), can be linked to three other concepts that are present in my outlook of data analytics (well related to learning attributes): machine learning, neural networks and deep learning. Again, these are all strong paths for data analytics. Machine learning, with coinage attributed to Arthur Samuel in 1959, is related to computers and the ability to learn without being explicitly programmed. Neural networks, or as Nielsen (2015) puts, "beautiful biologically-inspired programming paradigm which enables a computer to learn from observational data", have also been around for decades. Deep learning, or "a powerful set of techniques for learning in neural networks" (Nielsen, 2015), is set to bring machine learning closer to AI. Transformation takes time, as we see the seeds of all these ideas going back several decades.

A closer look at Andrew Ng's lab (deep learning and unsupervised feature learning) at Stanford (ufldl.stanford.edu) unveils a vast amount of related techniques, including:

a supervised learning and optimisation (linear regression, logistic regression, vectorisation, gradient checking, softmax regression, bias and variance, optimisers and objectives)

b supervised neural networks (multi-layer neural networks)

c supervised convolutional neural network (feature extraction, pooling, stochastic gradient descent, convolutional neural network)

d unsupervised learning (autoencoders, PCA whitening, sparse coding, independent component analysis

e self-taught learning.

All these are examples of the complexity behind an autonomous learning technology. However, in my opinion, it clearly shows a path to the transformation aiming at a better combination of human-machine approach to new problems.

All starts with a good explanation of the way we process structured and unstructured data in order to overcome problems, even complex ones (see how Schroeder, 2011, compared expert and non-expert metacognitive strategies for problem solving in calculus). In addition, encapsulating this into technology is a real challenge. AI building itself autonomously from massive amounts of data, cycle after cycle, represents a sound concept, but still requiring years of research. Technically, we may accept the current existence of narrow AI (NAI) and its many applications, with steps towards artificial general intelligence (AGI) and the futuristic artificial super-intelligence (ASI) as posed by Barrat (2013).

Let us get back to emotions, briefly. Assuming a cognitive-based decision making model, Bechara et al. (2000) explored that "cognitive operations, regardless of their content, depend on support processes such as attention, working memory and emotion" (p.295). They conclude, based on evidence from somatic markers, that "individuals make judgments not only by assessing the severity of outcomes and their probability of occurrence, but also and primarily in terms of their emotional quality" (p.305). Emotional and rational approaches, again, are considered altogether.

De Botton (2015) recently explored artificial emotional intelligence (AEI), acknowledging the expansion in the AI field, while adding emotions to the equation. It is 
not only about emotions, but how and when to show them, from an AI standpoint. Shopping represents a potential AEI application, in his assessment, as AEI 'will mean encoding consumer intelligence'.

But, with all that said, is the cat dead or alive? Invoking Schrödinger's famous paradox in quantum mechanics, we see quantum computing challenging the digital approach (where two electric distinct states are represented by 0 or 1 , the bit) and dives into the superposition of states with its qubit ( 0 and 1 simultaneously). The main concept I want to stress here is the challenge of a system (i.e., Sumerians and the evolution of numeric systems), which is the prevailing frame of reference for measuring and computing: moving beyond the digital logic (Lopata, 2013). Google's D-wave quantum computing is just one example of applications of such technology.

\section{Future of data analysis}

In 1926 Tesla stated:

"When wireless is perfectly applied the whole earth will be converted into a huge brain, which in fact it is, all things being particles of a real and rhythmic whole. We shall be able to communicate with one another instantly, irrespective of distance. Not only this, but through television and telephony we shall see and hear one another as perfectly as though we were face to face, despite intervening distances of thousands of miles; and the instruments through which we shall be able to do his will be amazingly simple compared with our present telephone. A man will be able to carry one in his vest pocket."

Predicting the future is a difficult task, but some can envision the future by dealing with trends.

Although clearly a field in expanding demand, it is hard to predict the future of data analysis, but my main argument here is to consider two paths:

a techniques

b human enhancement.

By techniques I consider a bold evolution in means of making sense of data, primarily by new ways of identifying, collecting, extracting, actually analysing, and presenting them (including on-demand), with an emphasis on less structured decisions. By human enhancement, I foresee a massive disruption in the role of humans and machines, especially in sensing and data analysis, having them working really together, seeking the best in each, not as the existing master slave relationship. Thanks to an ever-increasing access to computational power and memory, new potential is always available. The balance of these two dimensions will lead to a solid shift in this field.

Simply put, traditional techniques will not be able to solve the next needs of organisations and society. So, we will certainly do what we have been doing for ages: evolve. 


\section{References}

Barrat, J. (2013) Our Final Invention, St. Marteen's Press, New York.

Bechara, A., Damario, H. and Damasio, A.R. (2000) 'Emotion, decision making and the orbitofrontal cortex', Cerebral Cortex, Vol. 10, No. 3, pp.295-307.

Campbell, D.T. (1998) 'The experimenting society', in Dunn, W.N. (Ed.): The Experimenting Society: Essays in Honor of Donald T. Campbell, pp.35-68, Transaction Publishers, New Brunswick, NJ.

Chen, H., Chiang, R.H. and Storey, V.C. (2012) 'Business intelligence and analytics: from big data to big impact', MIS Quarterly, Vol. 36, No. 4, pp.1165-1188.

Clarke, A. (1980) 'Electronic tutors', Omni Magazine, June.

Damasio, A. (1996) Descartes' Error: Emotion, Reason, and the Human Brain, Putnam, London/UK.

De Botton, A. (2015) Six Areas that Artificial Emotional Intelligence will Revolutionise, Wired Magazine, November, Condé Nast, San Francisco, CA.

Dreyfus, H.L. and Dreyfus, S.E. (1986) Mind over Machine: The Power of Human Intuition and Expertise in the Era of the Computer, Free Press, New York.

George, G., Haas, M.R. and Pentland, A. (2014) 'From the editors: big data and management', Academy of Management Journal, Vol. 57, No. 2, pp.321-326.

Gibson, J.J. (1977) 'The theory of affordances', in Shaw, R. and Bransford, J. (Eds.): Perceiving, Acting, and Knowing, pp.67-82, Lawrence Erlbaum, Hillsdale, NJ.

IMA and ACCA (2013) Big Data: Its Power and Perils, ACCA, London/UK.

Kaplan, R. (1999) The Nothing that Is: The Natural History of Zero, Oxford, New York.

Kurzweil, R. (2012) How to Create a Mind, Penguin, New York.

Lerner, J.S., Li, Y., Valdesolo, P. and Kassam, K.S. (2015) 'Emotion and decision making', Annual Review of Psychology, Vol. 66, pp.799-823.

Lopata, P. (2013) 'Beyond digital: a brief introduction to quantum computing', The Next Wave, Vol. 20, No. 2, pp.33-37.

Lowenstein, G. and Lerner, J.S. (2003) 'The role of affect in decision making', in Davidson, R., Scherer, K. and Goldsmith, H. (Eds.): Handbook of Affective Science, pp.619-642, Oxford University Press, New York.

McCarthy, J. (2007) What is Artificial Intelligence? [online] http://www-formal.stanford.edu/jmc/whatisai/whatisai.html (accessed 6 May 2016).

National Transportation Safety Board (NTSB) (2008) Safety Recommendation A-11-028, NTSB, Washington, DC.

Nielsen, M.A. (2015) 'Neural Networks and Deep Learning, Determination Press [online] http://www.neuralnetworksanddeeplearning.com (accessed 6 May 2016).

Office of the Federal Register (2012) 14 Code of Federal Regulations: Aeronautics and Space (Takeoff Speeds, 25.107), US Government Printing Office, Washington, DC.

Pease, G., Beresford, B. and Walker, L. (2014) Developing Human Capital, Wiley, New Jersey.

Samuel, A. (1959) 'Some studies in machine learning using the game of checkers', IBM Journal of Research and Development, Vol. 3, No. 3, pp.210-229.

Schroeder, L.B. (2011) Investigating a Metacognitive Strategy for Solving Indefinite Integration Problems in Calculus: An fMRI Study, U. of Connecticut, Unpublished PhD Dissertation, UMI 3485447.

Tesla, N. (1926) 'When woman is boss.', Colliers, 30 January.

Wiegmann, D.A. and Shappell, S.A. (2003) A Human Error Approach to Aviation Accident Analysis, Ashgate, Burlington, VT. 